\title{
Análise de citações dos trabalhos da Compós 2008
}

\author{
Alex Primo, Ida Stumpf, \\ Gilberto Consoni e Stefanie Carlan da Silveira
}

\section{Resumo}

A citação é uma prática utilizada na produção de textos científicos que tem como função primária correlacionar dois textos. Como ferramenta dos estudos bibliométicos, a Análise de Citações (AC) permite relacionar 0 citante com 0 citado, mostrando as fontes que 0 primeiro utilizou para produzir seu trabalho. Embora apresente limitações, a análise de citações pode contribuir para entender a estrutura e 0 desenvolvimento de uma ciência, bem como as regularidades do comportamento dos cientistas no uso de fontes de informação. Este trabalho apresenta uma análise de citações da totalidade de trabalhos aprovados pelos Grupos de Trabalho (GT) do XVII Encontro da Associação Nacional dos Programas de Pós-Graduação em Comunicação Compós 2008. A partir dos dados coletados, buscou-se observar: autores, títulos, tipos de publicação (livros, capítulos, periódicos, outros), periódicos mais citados, freqüência de trabalhos coletivos, etc. Entre outros cruzamentos, apresenta-se um comparativo entre as citações a autores nacionais e estrangeiros.

\section{Palavras-chave}

Análise de citações. Compós. Bibliometria. Comunicação.

\section{Alex Primo | alex.primo@terra.com.br}

Doutor em Informática na Educação pela Universidade Federal do Rio Grande do Sul - UFRGS. Professor do Departamento de Comunicação e do Programa de Pós-Graduação em Comunicação e Informação da UFRGS. Pesquisador do CNPq.

\section{Ida Stumpf I irstumpf@ufrgs.br}

Doutora em Ciências da Comunicação pela Universidade de São Paulo - USP. Professora titular do Departamento de Ciências da Informação e do Programa de Pós-Graduação em Comunicação e Informação da UFRGS. Pesquisadora do CNPq.

Gilberto Consoni I gilberto@consoni.com.br

Mestrando em Comunicação e Informação pela UFRGS.

Stefanie Carlan da Silveira | fanics@gmail.com Mestranda em Comunicação e Informação pela UFRGS.

\section{Introdução}

A citação é uma prática utilizada na produção de textos científicos que tem como função primária correlacionar dois textos. Como ferramenta dos estudos bibliométicos, a Análise de Citações (AC) permite relacionar o citante com 0 citado, mostrando as fontes que o primeiro utilizou para produzir seu trabalho. Embora apresente limitações, a análise de citações pode contribuir para entender a estrutura e o desenvolvimento de uma ciência, bem como as regularidades do comportamento dos cientistas no uso de fontes de informação. Este trabalho apresenta uma análise de citações da totalidade de trabalhos aprovados pelos Grupos de Trabalho (GT) do XVII Encontro da Associação Nacional dos Programas de Pós-Graduação em Comunicação - Compós 2008. A partir dos dados coletados, buscou-se observar: autores, títulos, tipos de publicação (livros, capítulos, periódicos, outros) e periódicos mais citado e autoria dos trabalhos. Entre outros cruzamentos, apresenta-se um comparativo entre as citações a autores nacionais e estrangeiros. 


\section{$2 A$ análise de citações}

Citar é 0 ato de remeter um documento para outro. Esta ação tem como função básica promover a correlação entre dois trabalhos, um que cita e outro que é citado. A análise de citações de trabalhos científicos é um recurso da bibliometria que estuda as relações entre 0 citante e 0 citado, mostrando em quais fontes 0 autor se valeu para realizar seu estudo, dando autoria às idéias que apresenta e que não são originais. A referência a estas obras é mencionada em uma lista de referências, no final dos trabalhos. Este método, segundo Sanz Casado (1994), permite conhecer os hábitos de informação de determinados grupos de usuários, devido ao costume que têm os pesquisadores em apoiar-se em trabalhos publicados anteriormente para produzir seus próprios trabalhos.

A análise das referências é um método aplicado ao estudo de comunidades de pesquisadores, tendo em vista que são eles que utilizam as fontes formais, principalmente os livros e os periódicos, para fundamentar seus trabalhos e que publicam seus resultados para serem lidos e também citados por outros pesquisadores.

Hoje, a cultura das publicações está ligada à cultura das citações e uma publicação científica é facilmente reconhecida pelas referências a outros trabalhos científicos. Mensurar o número de vezes que um documento é citado nos trabalhos produzidos por uma determinada comunidade científica significa medir sua influência, seu impacto e sua qualidade para a pesquisa.

Entre os estudos que a análise das citações permite realizar está o dos padrões de citação. Estes estudos permitem inferir 0 comportamento dos pesquisadores em relação ao uso da literatura, mostrando as tendências de utilização por determinada corrente teórica ou metodológica, por publicações de determinado tipo, por documentos publicados em determinados países ou idiomas, como também identificar o uso mais intenso e a preferência por determinados títulos de periódicos. Entre os diversos autores que estudaram 0 assunto, Rosseau (1998) afirma que existe variação nos hábitos de citação dos autores nas diferentes áreas da ciência. Esta variação tanto pode ser em relação à idade das publicações que utilizam, à proximidade paradigmática, ao idioma das fontes utilizadas e outros aspectos que permeiam a produção do conhecimento.

Em relação à idade das publicações, uma das conclusões a que Velho (1986a) chegou foi de que as formas de distribuição da idade das citações variam entre as áreas científicas. Embora, de uma maneira geral, os cientistas precisam estar cientes apenas dos trabalhos recentes, Meadows (1999), ao referir-se especificamente aos humanistas, afirma que eles ainda continuam a citar a literatura antiga porque as idéias fundamentais que embasam estas áreas de conhecimento custam a mudar. São áreas em 
que as inovações não ocorrem com freqüência e, muitas vezes, os estudiosos têm na literatura antiga a matéria prima para suas investigações, portanto continuam citando os mesmos autores.

Outra descoberta de Velho (1986b) é de que os padrões de citação estão sempre associados ao passado educacional dos autores, ou seja, os hábitos adquiridos por um pesquisador na pós-graduação os acompanham durante sua vida profissional. Se os pesquisadores fizeram doutorado no exterior, é bem possível que as referências à literatura internacional sejam as mesmas dos livros e artigos que encontraram na biblioteca da instituição em que se titularam, ou que sejam as mesmas indicadas por seu orientador. E esse processo se perpetua, pois, quando no papel de orientadores, os pesquisadores indicarão a seus orientandos aqueles autores que conhecem e que representam os fundamentos do seu saber. Isto tem a ver também com 0 que autores como Lancaster, Burger e Rausfuss (1992) indicam como proximidade paradigmática, ou seja, quando a produção intelectual mostra a relação que um pesquisador ou um grupo de pesquisadores mantém com determinados autores. Vanz (2004), estudando o assunto, afirma que "a influência recebida de autores, teorias, paradigmas, metodologias, idiomas, bibliografias e tendências de pesquisa são incorporadas e transmitidas aos colegas e, de forma mais acentuada, aos alunos e aos orientandos que cercam o pesquisador" ( p. 37).
Nas práticas de citação, Moed e outros (1985) identificaram o fenômeno da citação doméstica (in-house citation) que consiste na tendência de citar os autores "da casa", ou seja, quando os pesquisadores utilizam, predominantemente, a literatura produzida pelos colegas de departamento ou de universidade. Esta prática pode indicar uma proximidade paradigmática, mas também pode também ser vista como uma limitação de conhecimento que não vai além das fronteiras da própria instituição, indicando um grau significativo de endogenia.

Vários fatores podem influenciar os autores na escolha das fontes para produzir seu trabalho. As razões destas escolhas ainda não foram suficientemente estudadas, mas Alvarenga (1998) indica que existem implicações de ordem psicológica, sociológica, política e histórica envolvidas no ato de citar. As de ordem psicológica dizem respeito aos hábitos e comportamentos já enraizados no sujeito na forma de produzir conhecimento e na hora de citar; as de cunho sociológico refere-se, principalmente, às escolhas comuns aos membros dos grupos de pesquisa que têm a ver com a sensação de pertencimento a determinada comunidade de pesquisadores; as de caráter político referem-se ao sentimento de poder do autor citante por demonstrar domínio por determinadas idéias ou autores, ou também de citar autores de determinada instituição por conveniência; e as implicações de origem históricas mantêm relação com as influências 
que 0 autor vem acumulando sobre utilização de determinados autores ou grupo ao longo do tempo.

Para Carvalho (1975, p.119), "não se pode esperar que todos os autores sejam cuidadosos, objetivos e conscientes no momento de mencionar suas fontes de informação. Alguns pecam por excesso, outros por omissão." Às vezes, 0 autor pode ter incorporado uma idéia que não identifica mais quem a introduziu no conjunto de saberes que ele detém; e, em outras, 0 autor pode ser tão escrupuloso que até mesmo as idéias de senso comum ele se omite de assumir a autoria. $0 u$, por vezes, cita muitos autores pensando mostrar erudição e domínio de um conteúdo.

A possibilidade de um documento ser citado depende de vários fatores, como o idioma em que está escrito, o tipo de material em que está registrado ou a data de sua publicação. Mas, mais do que tudo, os estudos de usuários de informação mostram que os pesquisadores usam o que está acessível, sendo um fato comprovado que só se cita o que está disponível (SANZ CASADO, 1994). Com a possibilidade das publicações eletrônicas terem acesso livre, hoje em dia, esta premissa pode ser trocada por outra que afirma que, se o texto não está disponível na Internet, não será citado. Estudos já confirmam que os documentos eletrônicos online são mais citados do que os impressos e esta é uma prática que está ficando cada vez mais freqüente (LAWRENCE, 2001).
Uma das práticas mais problemáticas para estudar as citações são as auto-citações, entendendo-se como tal aquelas em que os autores citam seus próprios trabalhos. No conjunto de todas as citações apresentadas num trabalho elas geralmente têm pouca representatividade, mas as razões de seu uso podem variar desde 0 narcisismo até a necessidade que os autores têm de mostrar que já vem pesquisando sobre determinado assunto há mais tempo. Embora as razões da auto-citação possam ser diferentes de autor para autor, o fato é que ao utilizá-la 0 autor dá visibilidade a seus trabalhos anteriores, beneficiando-se com esta divulgação. (FOWLER; AKSNES, 2007).

A contagem de citações, como único método para investigar 0 assunto, deve ser utilizada de modo crítico, cuidadoso e dentro de um contexto. Daí porque, muitas vezes, a simples mensuração das referências e de seus elementos básicos se torna insuficiente para estudar o fenômeno das citações, devendo ser acompanhada de estudos qualitativos que mostrem o significado de determinadas práticas, de determinados autores e de determinados documentos para a pesquisa.

\section{Procedimentos metodológicos}

Para este estudo bibliométrico foram analisados os 120 artigos aprovados para apresentação no XVII Encontro da Associação Nacional dos Programas de Pós-Graduação em Comunicação, realizado na UNIP, em São Paulo, de 3 a 6 de junho de 2008. Para tanto, utilizou-se os arquivos 
em PDF dos respectivos trabalhos, disponíveis na seção "Biblioteca" do site da associação (http:// www.compos.org.br).

De posse dos documentos digitais, em uma planilha Excel foram anotadas todas as referências bibliográficas (incluindo aquelas em notas de rodapé) apresentadas em cada trabalho, com os respectivos autores, instituições (caso os autores fossem brasileiros), ano e tipo de publicação (livro, capítulo, artigo de periódico ou anais, e outros ${ }^{1}$ ). Durante esse processo, foram produzidas listas com todos os periódicos e eventos citados. Para cada trabalho registrou-se também os nomes dos autores e suas instituições (quando brasileiros), conforme os dados disponíveis na plataforma Lattes.

Finalmente, este levantamento inicial apontou os casos de auto-citação, dado que, como ver-se-á, foi importante para a delimitação dos autores mais citados na Compós 2008.

Esta coleta encontrou 2111 referências nos 120 trabalhos analisados. A planilha completa foi então submetida a um tratamento estatístico, que efetuou diferentes cruzamentos e quantificações, que são relatados e discutidos a seguir.

\section{Análise e discussão dos dados}

Dos 120 artigos aprovados, 92 foram escritos por apenas um pesquisador (76,6\%), 26 por 2 autores $(21,6 \%)$ e apenas 2 por 3 autores (1,6\%). Esse dado mostra que os pesquisadores da área pouco desenvolvem trabalhos em co-autoria. Ou seja, os papers da Compós 2008 relatam principalmente investigações individuais, evidenciando poucos esforços colaborativos de pesquisa.

Em 2008, registrou-se 2111 referências bibliográficas (incluídas as auto-citações), sendo que um trabalho do GT Comunicação e Cultura não apresentou nenhuma citação. Dentre os trabalhos citados, 1850 (87,6\%) são assinados por apenas um autor; $220(10,4 \%)$ apresentam 2 autores; 33 (1,6\%), 3 autores; 5 (0,2\%), 4 autores; e um trabalho apresentou co-autoria de 6 pesquisadores. Quanto à nacionalidade, 1321 trabalhos $(62,58 \%)$ foram escritos por autores estrangeiros, $783(37,09 \%)$ por autores nacionais, 7 trabalhos combinavam autores nacionais e estrangeiros (essa modalidade é apresentada na tabela a seguir como "misto").

Separando-se o número de referências por GT, verificou-se que os trabalhos dos GTs "Comunicação e Cibercultura" e "Recepção, Usos e Consumo Midiáticos" foram os que incluíram o maior número de fontes bibliográficas: em média, 22,1 e 21,2 referências por trabalho, respectivamente. No extremo oposto, os GTs de "Epistemologia da Comunicação" e "Fotografia, Cinema e Vídeo" foram os que apresentaram 0 menor número: médias de 14,4 e 11,9. A tabela 1 detalha tais valores. 
Tabela 1 - Quantidade de trabalhos referenciados por GT e nacionalidade dos autores citados

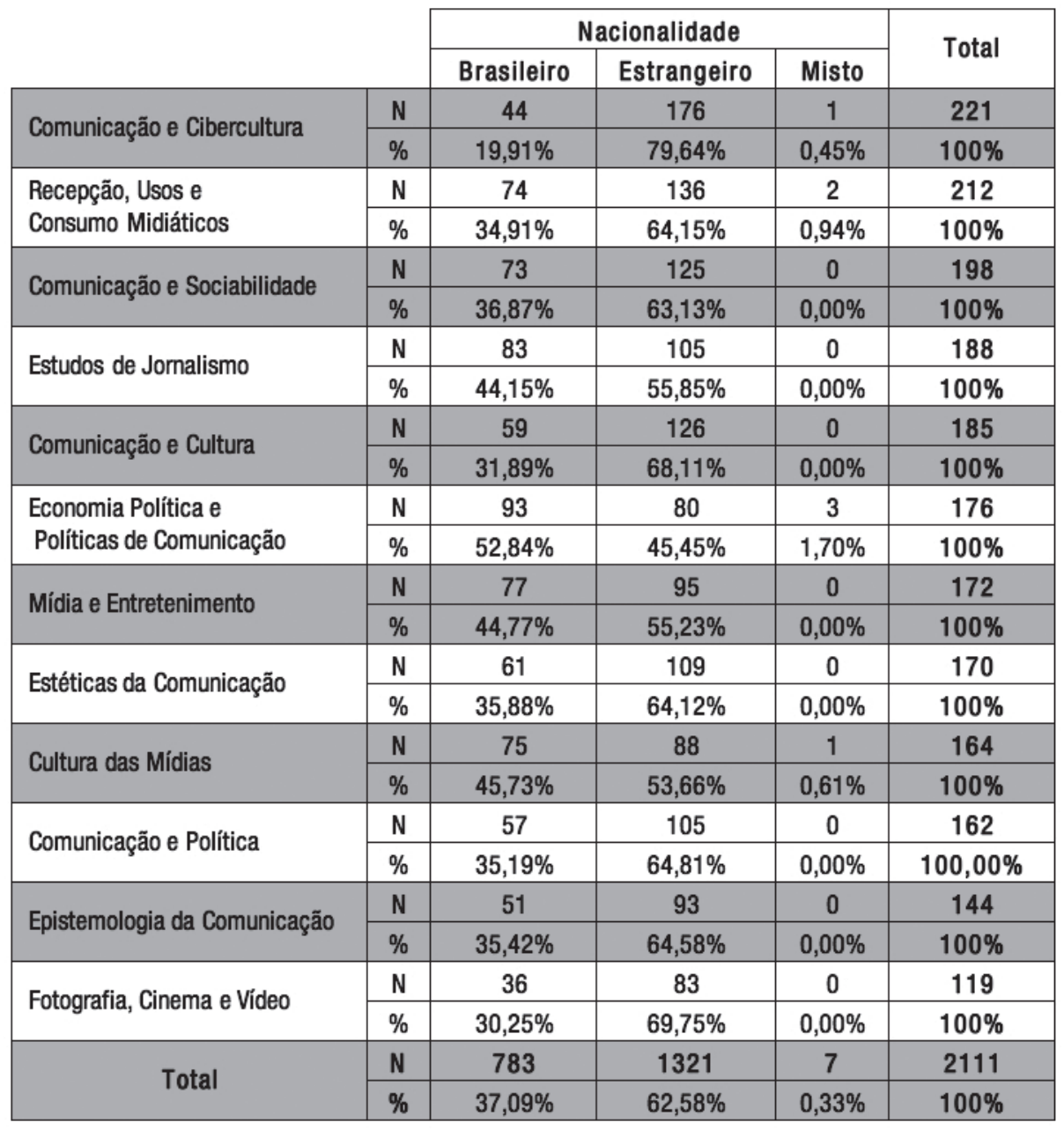


A mesma tabela discrimina a porcentagem de citações a autores nacionais e estrangeiros. Constata-se que, apesar de grande produção em cibercultura no país, existe pouca citação entre os pares da comunidade nacional: quase $80 \%$ das citações referem-se a pesquisadores estrangeiros. Os GTs de Estudos em Jornalismo, Cultura das Mídias e Economia Política e Políticas de Comunicação apresentam melhor equilíbrio. Este último é o único que cita mais autores nacionais $(52,84 \%)$ do que estrangeiros.

Os trabalhos da Compós 2008 confirmam um padrão da área, o uso de livros como principal fonte bibliográfica: 1217 (57,7\%) livros e 275 (13\%) capítulos de livros foram referenciados. Foram também encontradas 297 $(14,1 \%)$ citações a artigos de periódicos e 107 $(10,2 \%)$ a trabalhos em anais. Finalmente, 215 $(10,2 \%)$ citações foram classificadas na grande categoria "outros", abarcando materiais como jornais, revisas, sites, vídeos, etc. Meadows (1999) confirma que trata-se de uma tradição das Ciências Sociais o uso mais intenso da publicação em formato de livro, diferentemente das chamadas ciências duras.

Entre os eventos citados (congressos, simpósios, etc.), a própria Compós foi a mais citada (41 referências, 40,6\%), seguida pela Intercom (26 citações, $25,7 \%$ ). Os valores comprovam a relevância desses eventos na área. 0 eventos seguintes mais citados foram referenciados apenas 2 vezes: ABRALIC, SBPJor, Forumdoc, Hawaii International Conference on System Sciences, Annual Workshop on the Weblogging Ecosystem. Os outros eventos obtiveram apenas uma referência. É importante lembrar que muitos trabalhos registrados em anais são eventualmente publicados em periódicos. Logo, é possível que um mesmo texto tenha sido referenciado em um GT como artigo de periódico e em outro como trabalho em anais. Esta situação, no entanto, não pôde ser discriminada nesta pesquisa.

A tabela 2 lista os periódicos mais citados e sua classificação no sistema Qualis² . A listagem apresenta apenas os periódicos com mais de duas citações. A E-Compós, revista científica da mesma associação que promove 0 evento pesquisado neste trabalho, teve quase 3 vezes mais citações que aqueles que ocupam a segunda colocação no ranking. Pode-se supor que a E-Compós possui tal penetração não apenas por sua qualidade, mas também por ser um periódico online de acesso livre. De toda forma, a hipótese só poderá ser confirmada a partir de outros estudos. Mesmo assim, podese inferir que 0 alto número de referências à E-Compós não ocorre pelo simples fato do evento analisado ser promovido pela mesma associação que publica aquele periódico. 
Tabela 2 - Periódicos mais citados

\begin{tabular}{|c|c|c|c|}
\hline \multirow{2}{*}{ Periódico } & \multirow{2}{*}{ N } & \multicolumn{2}{|c|}{ Qualis $^{3}$} \\
\hline & & 2008 & 2009 \\
\hline $\begin{array}{c}\text { E-Compós - } \\
\text { Revista da Compós }\end{array}$ & 16 & A & B2 \\
\hline Revista da Famecos & 8 & A & B2 \\
\hline $\begin{array}{l}\text { Revista Comunicação, } \\
\text { Midia e Consumo }\end{array}$ & 6 & A & B2 \\
\hline Revista LIBERO & 5 & B & B3 \\
\hline $\begin{array}{l}\text { American Journal } \\
\text { of Sociology }\end{array}$ & 5 & B & \\
\hline $\begin{array}{l}\text { Journal of computer-mediated } \\
\text { communication }\end{array}$ & 5 & & \\
\hline Revista Contracampo & 4 & A & B2 \\
\hline Revista Cinética & 4 & & \\
\hline Fronteiras, Estudos Midiáticos & 4 & A & B3 \\
\hline $\begin{array}{l}\text { Revista Latinoamericana } \\
\text { de Comunicación }\end{array}$ & 4 & B & B2 \\
\hline Revista ECO-PÓS & 4 & B & B4 \\
\hline Media, Culture \& Society & 4 & A & A2 \\
\hline $\begin{array}{l}\text { Revista Brasileira } \\
\text { de História }\end{array}$ & 3 & A & B2 \\
\hline $\begin{array}{c}\text { Revista Brasileira } \\
\text { de Ciências da Comunicação }\end{array}$ & 3 & A & B2 \\
\hline $\begin{array}{c}\text { Contemporanea: } \\
\text { Revista de Comunicação } \\
\text { e Cultura }\end{array}$ & 3 & B & B3 \\
\hline American Sociological Review & 3 & & \\
\hline Cadernos de Televisão & 3 & & \\
\hline $\begin{array}{l}\text { Revista Novas Tecnologias } \\
\text { na Educação (RENOTE) }\end{array}$ & 3 & C & B5 \\
\hline Estudos Históricos & 3 & A & B1 \\
\hline Journalism Quarterly & 3 & & \\
\hline Newspaper Research Journal & 3 & & \\
\hline $\begin{array}{l}\text { Revista Administração } \\
\text { de Empresas }\end{array}$ & 3 & C & B4 \\
\hline $\begin{array}{c}\text { Annual Review of Clinical } \\
\text { Psychology }\end{array}$ & 3 & & \\
\hline $\begin{array}{c}\text { Revista dos Transportes } \\
\text { Públicos }\end{array}$ & 3 & A & B3 \\
\hline Revista de Cultura Vozes & 3 & A & B1 \\
\hline American Economic Review & 3 & A & \\
\hline Revista Eptic & 3 & A & B4 \\
\hline $\begin{array}{c}\text { Cognitio: } \\
\text { Revista de Filosofia }\end{array}$ & 2 & C & B4 \\
\hline Atlantic Monthly & 2 & & \\
\hline Nature & 2 & A & A1 \\
\hline
\end{tabular}

\begin{tabular}{|c|c|c|c|}
\hline \multirow{2}{*}{ Periódico } & \multirow{2}{*}{ N } & \multicolumn{2}{|c|}{ Qualis } \\
\hline & & 2008 & 2009 \\
\hline Webology & 2 & & \\
\hline Surveillance \& Society & 2 & C & B1 \\
\hline Ciberlegenda & 2 & c & B5 \\
\hline Communications & 2 & & \\
\hline Significação. & 2 & A & B2 \\
\hline $\begin{array}{l}\text { Art et Mode: } \\
\text { attirance et divergence }\end{array}$ & 2 & & \\
\hline Revue Réseaux & 2 & & \\
\hline Revista Galáxia & 2 & A & B2 \\
\hline UNIrevista & 2 & C & B5 \\
\hline Journal of Communication & 2 & A & \\
\hline Revista Matrizes & 2 & & B3 \\
\hline Frames per Second & 2 & & \\
\hline Sight and Sound & 2 & & \\
\hline Diálogos de la Comunicación & 2 & B & $\mathrm{A} 2$ \\
\hline Diálogos Possiveis & 2 & B & B5 \\
\hline Hermés & 2 & A & B1 \\
\hline $\begin{array}{l}\text { Revista Latinoamericana } \\
\text { de Estudios Del Discurso }\end{array}$ & 2 & B & B1 \\
\hline Revista da ESPM & 2 & C & B5 \\
\hline Revista Margem & 2 & B & B4 \\
\hline Cahiers du Cinéma & 2 & C & A2 \\
\hline Journalism & 2 & B & \\
\hline $\begin{array}{l}\text { European Journal } \\
\text { of Communication }\end{array}$ & 2 & & \\
\hline Party Politics & 2 & A & \\
\hline Lua Nova & 2 & A & B1 \\
\hline $\begin{array}{l}\text { International Journal } \\
\text { of Qualitative Methods }\end{array}$ & 2 & & \\
\hline Revista InTexto & 2 & A & B3 \\
\hline $\begin{array}{l}\text { Revista Comunicação } \\
\text { e Sociedade }\end{array}$ & 2 & A & B3 \\
\hline Chasqui & 2 & C & B1 \\
\hline Sala de Prensa & 2 & & \\
\hline- & - & - & - \\
\hline
\end{tabular}


Através dessa listagem, observa-se que os

periódicos nacionais de comunicação mais

citados possuem a classificação Qualis A e B

Nacional. Por outro lado, confirma-se também 0

caráter interdisciplinar da área, tendo em vista as referências a outros campos como Educação,

Administração, História, Economia, etc.

Houve grande dispersão quanto aos títulos individuais (livros, artigos, etc.) mais citados. A tabela 3 exibe os resultados com mais de 3 referências.

Tabela 3 - Títulos mais citados

\begin{tabular}{|c|c|c|}
\hline Título & Autor & N \\
\hline Dos meios às mediações: comunicação, cultura e hegemonia & MARTIN -BARBERO, Jesus & 8 \\
\hline $\begin{array}{l}\text { A condição pós-moderna: } \\
\text { uma pesquisa sobre as origens da mudança cultural }\end{array}$ & HARVEY, David & 5 \\
\hline A identidade cultural na pós-modernidade & HALL, Stuart & 5 \\
\hline A sociedade em rede: e era da informação. Economia, sociedade e cultura & CASTELLS, Manuel & 5 \\
\hline Consumidores e cidadãos: conflitos multiculturais da globalização & CANCLINI, Nestor Garcia & 5 \\
\hline Marxismo e filosofia da linguagem & BAKHTIN, Mikhail & 5 \\
\hline 0 local da cultura & BHABHA, Homi K. & 5 \\
\hline $\begin{array}{l}\text { A cultura da mídia - estudos culturais: } \\
\text { identidade e política entre o moderno e o pós-moderno }\end{array}$ & KELLNER, Douglas & 4 \\
\hline A sociedade do espetáculo & DEBORD, Guy & 4 \\
\hline Antropológica do espelho: uma teoria da comunicação linear e em rede & SODRÉ, Muniz & 4 \\
\hline $\begin{array}{l}\text { As tecnologias da inteligência: o futuro do pensamento na era da } \\
\text { informática }\end{array}$ & LÉVY, Pierre & 4 \\
\hline Cineastas e imagens do povo & BERNARDET, Jean-Claude & 4 \\
\hline Vigiar e punir: nascimento da prisão & FOUCAULT, Michel & 4 \\
\hline A imagem-tempo & DELEUZE, Gilles & 3 \\
\hline A invenção do cotidiano & CERTEAU, Michel de & 3 \\
\hline A invenção do Nordeste e outras artes & ALBUQUERQUE JR, Durval Muniz & 3 \\
\hline A metrópole e a vida mental. & SIMMEL, George & 3 \\
\hline A mídia e a modernidade: uma teoria social da mídia & THOMPSON, John B. & 3 \\
\hline A sociedade enfrenta sua mídia: dispositivos sociais de crítica mediática & BRAGA, José Luiz & 3 \\
\hline As palavras e as coisas: uma arqueologia das ciências humanas & FOUCAULT, Michel & 3 \\
\hline Da diáspora: identidades e mediações culturais & HALL, Stuart & 3 \\
\hline História da sexualidade i: a vontade de saber & FOUCAULT, Michel & 3 \\
\hline Imagined communities: Reflexions on the origins and spread of nationalism & ANDERSON, Benedict & 3 \\
\hline Mídia, teoria e política & LIMA, Venício Artur de & 3 \\
\hline Modernidade líquida & BAUMAN, Zygmunt & 3 \\
\hline Nação e consciência nacional & ANDERSON, Benedict & 3 \\
\hline 0 espaço crítico & VIRILIO, Paul & 3 \\
\hline 0 espírito comum: comunidade, mídia e globalismo & PAIVA, Raquel & 3 \\
\hline 0 pós-moderno explicado às crianças & LYOTARD, Jean-François & 3 \\
\hline Para entender as notícias: linhas de análise do discurso jornalístico & PONTE, Cristina & 3 \\
\hline Por que estudar a mídia? & SILVERSTONE, Roger & 3 \\
\hline Pós-modernismo: a lógica cultural do capitalismo tardio & JAMESON, Frederic & 3 \\
\hline
\end{tabular}


Ao analisar-se os autores mais citados, encontrouse 205 auto-citações ${ }^{4}$, o que representa quase 10\% do total de 2111 referências da Compós 2008. Este fato alterou o ranking dos autores mais citados no evento. Ao se descontar as auto-citações, alguns pesquisadores que estavam entre os mais citados na listagem inicial passaram a ocupar posições mais distantes do topo do ranking ou até deixaram de figurar. Realizada a reclassificação, os autores com mais de 5 citações foram:

Tabela 4 - Autores mais citados

\begin{tabular}{|l|c|}
\hline \multicolumn{1}{|c|}{ Autores } & Citações \\
\hline DELEUZE, Gilles & 26 \\
\hline FOUCAULT, Michael & 24 \\
\hline CANCLINI, Nestor Garcia & 19 \\
\hline HALL, Stuart & 16 \\
\hline MARTÍN-BARBERO, Jesus & 16 \\
\hline BAUMAN, Zygmunt & 15 \\
\hline BOURDIEU, Pierre & 15 \\
\hline GUATTARI, Félix & 15 \\
\hline BAUDRILLARD, Jean & 11 \\
\hline SODRE, Muniz & 10 \\
\hline BAKHTIN, Mikhail & 10 \\
\hline BENJAMIM, Walter & 10 \\
\hline BERNARDET, Jean Claude & 10 \\
\hline CASTELLS, Manuel & 10 \\
\hline ECO, Umberto & 10 \\
\hline MACHADO, Arlindo & 10 \\
\hline LOPES, Maria Immacolata Vassalo & 9 \\
\hline BRAGA, José Luiz & 9 \\
\hline VIRILIO, Paul & 9 \\
\hline BLUMER, Herbert & 8 \\
\hline GUMBRECHT, Hans Ulrich & 8 \\
\hline JAMESON, Frederic & 8 \\
\hline LÉYY, Pierre & 8 \\
\hline ORTIZ, Renato & 8 \\
\hline SANTAELLA, Lucia & 8 \\
\hline VERÓN, Eliseo & \\
\hline
\end{tabular}

\begin{tabular}{|l|c|}
\hline \multicolumn{1}{|c|}{ Autores } & Citaçöes \\
\hline FIORIN, José Luiz & 7 \\
\hline HARVEY, David & 7 \\
\hline LOTMAN, Yuri & 7 \\
\hline MARX, Karl & 7 \\
\hline MELO, José Marques de & 7 \\
\hline PEIRCE, Charles Sanders & 7 \\
\hline SCHWARTZ, Yves & 7 \\
\hline MARTINO, Luiz Claudio & 6 \\
\hline BRITTOS, Valério Cruz & 6 \\
\hline PRIMO, Alex & 6 \\
\hline ANDERSON, Benedict & 6 \\
\hline BARTHES, Roland & 6 \\
\hline BERGSON, Henri & 6 \\
\hline BHABHA, Homi & 6 \\
\hline DEWEY, John & 6 \\
\hline FLUSSER, Vilém & 6 \\
\hline KELLNER, Douglas & 6 \\
\hline LIMA, Venício de Araújo & 6 \\
\hline MCLUHAN, Marshall & 6 \\
\hline NIETZSCHE, Friedrich & 6 \\
\hline SANTOS, Boaventura de Sousa & 6 \\
\hline STAM, Robert & 6 \\
\hline TRAQUINA, Nelson & 6 \\
\hline BOLAÑO, César Ricardo Siqueira & 5 \\
\hline PIEDRAS, Elisa & 5 \\
\hline PEREIRA, Vinicius Andrade & 5 \\
\hline BRASIL, André & \\
\hline
\end{tabular}

Um trabalho em co-autoria onde 2 autores citam outro trabalho produzido por ambos foi contado como 2 auto-citações. Se esse procedimento não for adotado, contabiliza-se 196 auto-citações. 
0s pesquisadores que apresentaram o maior número de auto-citações em seus trabalhos na Compós 2008 foram:

Tabela 5 - Auto-citações

\begin{tabular}{|l|r|}
\hline STEINBERGER, Margarethe Born & 13 \\
\hline CAIAFA, Janice & 8 \\
\hline FAUSTO NETO, Antônio & 7 \\
\hline BOLAÑO, César Ricardo Siqueira & 6 \\
\hline TRINDADE, Eneus & 6 \\
\hline PIEDRAS, Elisa & 5 \\
\hline FELDMAN, llana & 5 \\
\hline HENN, Ronaldo Cesar & 5 \\
\hline HERSCOVICI, Alain & 5 \\
\hline RECUERO, Raquel & 4 \\
\hline PEREIRA, Vinicius Andrade & 4 \\
\hline TRIVINHO, Eugênio & 4 \\
\hline DANTAS, Marcos & 4 \\
\hline FÍGARO PAULINO, Roseli Aparecida & 4 \\
\hline CASTRO, Gisela & 4 \\
\hline ROCHA, Rose de Melo & 3 \\
\hline
\end{tabular}

Este trabalho não tinha elementos para avaliar as razões para a auto-citação. Pode-se inferir, contudo, que elas podem ser usadas para demonstrar a continuidade de um projeto de pesquisa ou mesmo remeter o leitor a trabalhos anteriores, onde conceitos e dados do mesmo autor são discutidos com maior profundidade. Contudo, trabalhos qualitativos futuros são necessários para testar essas hipóteses.

Os dados quantitativos revelaram que $42,5 \%$ das referências foram publicadas nos 5 anos (20032007) que antecediam o deadline para submissão de trabalhos (30 de janeiro de 2008). Apesar dessa data limite, observou-se que 8 trabalhos de 2008 $(0,4 \%)$ foram referenciados. Considerando-se 0 dados quantitativos sobre as datas de publicação das referências, a listagem dos títulos (tabela 3) e autores mais citados (tabela 4), pode-se constatar que existe um equilíbrio entre fontes recentes e trabalhos "clássicos".

É importante destacar que os anos de publicação registrados na pesquisa referem-se àqueles mencionados pelos autores dos trabalhos aceitos para a Compós 2008. Logo, é possível que uma mesma referência bibliográfica tenha sido registrada com datas diferentes em 2 ou mais trabalhos. Além disso, como o padrão de referenciação utiliza a data da edição que o pesquisador possui, e não da primeira edição publicada, frequentemente um trabalho clássico publicado há várias décadas é referenciado com uma data recente. Estas circunstâncias acarretam alguns vieses nos dados quantitativos aqui relatados. 


\section{Breve comparativo histórico}

Como a análise de citações dos trabalhos da Compós está sendo pela primeira vez estudada e discutida ${ }^{5}$, não se pode ainda realizar um estudo longitudinal sobre a evolução dos padrões de referenciação da área. Logo, este trabalho, mesmo não tendo foco comparativo, apresenta a seguir um breve paralelo com um estudo anterior realizado por Samile Vanz, em 2004. A dissertação desta pesquisadora, hoje professora do departamento de Ciências da Informação da UFRGS, avaliou as 7.648 citações de 100 dissertações defendidas nos cursos de pós-graduação em Comunicação da UFRGS, PUCRS e UNISINOS, do Rio Grande do Sul, entre os anos de 1998 e 2000. Ainda que as amostras sejam bastante distintas quanto ao formato (dissertações X trabalhos de evento) e quanto à formação dos autores (mestrandos $\mathrm{X}$ doutores, na maioria), a comparação a seguir pode apontar indícios interessantes. Serão confrontados três indicadores que aparecem nas duas pesquisas: tipo de documento, citação por autor, citação por periódicos.

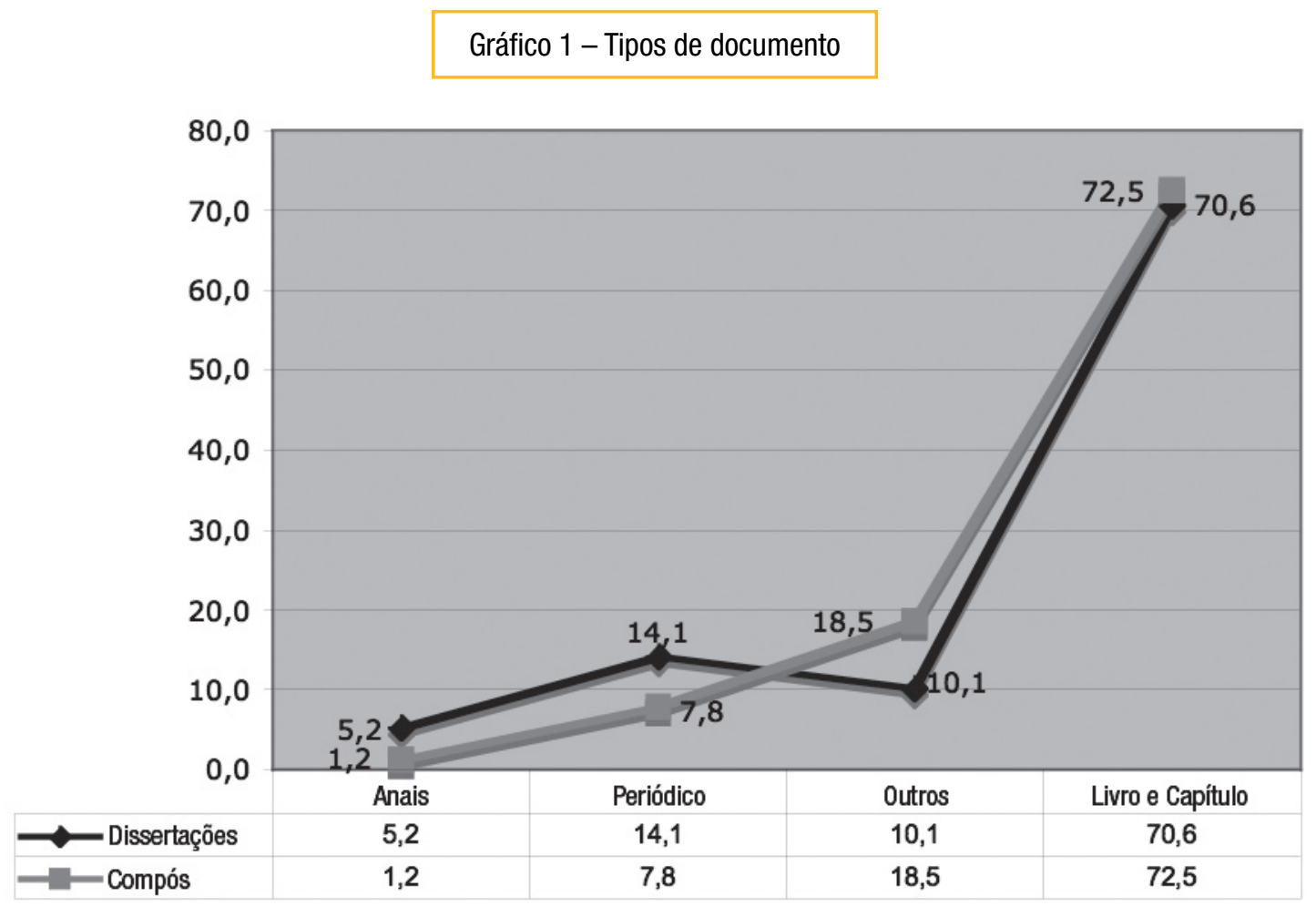

Além do presente trabalho sobre as citações nos trabalhos da Compós 2008, Maria Immacolata Vassallo de Lopes e Richard Romancini realizaram em paralelo um estudo sobre os artigos da Compós 2007. 
Os resultados das duas pesquisas apontam os livros

como principal fonte de referência, com pequena

variação. As citações de anais e de periódicos

sofreram uma variação mais significante, perdendo

espaço para a variável "outros".

Tabela 6 - Comparativo de periódicos citados

\begin{tabular}{|l|c|c|c|c|}
\hline \multirow{2}{*}{\multicolumn{1}{|c|}{ Periódicos }} & \multicolumn{2}{c|}{$\begin{array}{c}\text { Compós } \\
\text { 2008 }\end{array}$} & \multicolumn{2}{c|}{$\begin{array}{c}\text { Dissertações } \\
\text { Ey98-2000 }\end{array}$} \\
\cline { 2 - 5 } & N & $\%$ & N & $\%$ \\
\hline E-compós - Revista da Compós & 16 & 5,3 & - & - \\
\hline Revista da Famecos & 8 & 2,6 & 37 & 6,2 \\
\hline Comunicação, Mídia e Consumo & 6 & 2,0 & - & - \\
\hline American Journal of Sociology & 5 & 1,7 & - & - \\
\hline $\begin{array}{l}\text { Journal of computer-mediated } \\
\text { communication }\end{array}$ & 5 & 1,7 & - & - \\
\hline Líbero & 5 & 1,7 & - & - \\
\hline Revista Contracampo & 4 & 1,3 & - & - \\
\hline Revista Cinética & 4 & 1,3 & - & - \\
\hline Fronteiras, Estudos Midiáticos & 4 & 1,3 & - & - \\
\hline Latinoamericana de Comunicación & 4 & 1,3 & - & - \\
\hline Revista ECO-Pós & 4 & 1,3 & - & - \\
\hline Cultura Vozes & 3 & 1,0 & 13 & 2,2 \\
\hline Comunicação e Sociedade & 2 & 0,7 & 23 & 3,8 \\
\hline Diálogos de la Comunicación & 2 & 0,7 & 20 & 3,3 \\
\hline Telos & 1 & 0,3 & 20 & 3,3 \\
\hline Comunicação \& Política & 1 & 0,3 & 20 & 3,3 \\
\hline Screen & 1 & 0,3 & 19 & 3,2 \\
\hline Brasileira de Ciências Sociais & 1 & 0,3 & 16 & 2,7 \\
\hline $\begin{array}{l}\text { Estudios sobre las culturas } \\
\text { contemporáneas }\end{array}$ & 1 & 0,3 & 15 & 2,5 \\
\hline Journal of macromarketing & 1 & 0,3 & 12 & 2,0 \\
\hline Outros Periódicos & 225 & 74,3 & 403 & 67,4 \\
\hline Total & 303 & 100 & 598 & 100 \\
\hline
\end{tabular}

Foram selecionados os 10 periódicos mais citados de cada pesquisa. 0 único periódico que aparece nas 10 primeiras posições das duas pesquisas é a Revista Famecos. 0 periódico é o mais citado nas dissertações e ocupa a segunda posição nas referências dos trabalhos da Compós. Não fosse esta segunda colocação, poderíamos atribuir tal resultado por se tratar de um periódico que é do mesmo estado dos programas de pós-graduação pesquisados. Precisa, também, ser considerado que o periódico não existia na época desta primeira pesquisa e por isto não aparece nas duas. 
Tabela 7 - Comparativo de autores mais citados

\begin{tabular}{|c|c|c|c|c|}
\hline \multirow{2}{*}{ Autores } & \multicolumn{2}{|c|}{ Compós 2008} & \multicolumn{2}{|c|}{$\begin{array}{c}\text { Dissertações } \\
1998-2000\end{array}$} \\
\hline & $\mathrm{N}$ & $\%$ & $\mathrm{~N}$ & $\%$ \\
\hline DELEUZE, Gilles & 26 & 1,1 & 23 & 0,3 \\
\hline FOUCAULT, Michael & 24 & 1,0 & 53 & 0,7 \\
\hline CANCLINI, Nestor Garcia & 19 & 0,8 & 28 & 0,4 \\
\hline MARTÍN-BARBER0, Jesus & 16 & 0,7 & 28 & 0,4 \\
\hline HALL, Stuart & 16 & 0,7 & - & - \\
\hline BOURDIEU, Pierre & 15 & 0,6 & 67 & 0,9 \\
\hline BAUMAN, Zygmunt & 15 & 0,6 & - & - \\
\hline GUATTARI, Félix & 15 & 0,6 & - & - \\
\hline BAUDRILLARD, Jean & 11 & 0,5 & 51 & 0,7 \\
\hline ECO, Umberto & 10 & 0,4 & 83 & 1,1 \\
\hline MACHADO, Arlindo & 10 & 0,4 & 35 & 0,5 \\
\hline BENJAMIM, Walter & 10 & 0,4 & 34 & 0,4 \\
\hline BAKHTIN, Mikhail & 10 & 0,4 & - & - \\
\hline BERNARDET, Jean Claude & 10 & 0,4 & - & - \\
\hline CASTELLS, Manuel & 10 & 0,4 & - & - \\
\hline SODRÉ, Muniz & 10 & 0,4 & - & - \\
\hline $\begin{array}{l}\text { LOPES, Maria Immacolata } \\
\text { Vassalo }\end{array}$ & 9 & 0,4 & 30 & 0,4 \\
\hline BRAGA, José Luiz & 9 & 0,4 & - & - \\
\hline VIRILIO, Paul & 9 & 0,4 & - & - \\
\hline LÉVY, Pierre & 8 & 0,3 & 39 & 0,5 \\
\hline ORTIZ, Renato & 8 & 0,3 & 34 & 0,4 \\
\hline SANTAELLA, Lúcia & 8 & 0,3 & 20 & 0,3 \\
\hline BLUMER, Herbert & 8 & 0,3 & - & - \\
\hline GUMBRECHT, Hans Ulrich & 8 & 0,3 & - & - \\
\hline JAMESON, Frederic & 8 & 0,3 & - & - \\
\hline VERÓN, Eliseo & 8 & 0,3 & - & - \\
\hline MELO, José Marques de & 7 & 0,3 & 27 & 0,3 \\
\hline FAUSTO NETO, Antonio & 7 & 0,3 & 25 & 0,3 \\
\hline HARVEY, David & 7 & 0,3 & 21 & 0,3 \\
\hline FIORIN, José Luiz & 7 & 0,3 & - & - \\
\hline LOTMAN, Yuri & 7 & 0,3 & - & - \\
\hline MARX, Karl & 7 & 0,3 & - & - \\
\hline BHARTES, Roland & 6 & 0,2 & 81 & 1,0 \\
\hline MORIN, Edgar & 5 & 0,2 & 65 & 0,8 \\
\hline MAFFESOLI, Michel & 5 & 0,2 & 49 & 0,6 \\
\hline ADORNO, Theodor & 5 & 0,2 & 24 & 0,3 \\
\hline MATTELART, Armand & 4 & 0,2 & 41 & 0,5 \\
\hline RODRIGUES, Adriano & 3 & 0,1 & 20 & 0,3 \\
\hline CAPPARELLI, Sérgio & 1 & 0,04 & 39 & 0,5 \\
\hline MARCONDES FILHO, Ciro & 1 & 0,04 & 25 & 0,3 \\
\hline ORLANDI, Eni & 1 & 0,04 & 20 & 0,3 \\
\hline GREIMAS, Algirdas Julien & 0 & 0 & 39 & 0,5 \\
\hline FREUD, Sigmund & 0 & 0 & 35 & 0,5 \\
\hline HJELMSLEV, Louis & 0 & 0 & 26 & 0,3 \\
\hline GUARESCHI, Pedrinho & 0 & 0 & 22 & 0,3 \\
\hline OUTROS & 2032 & 84,14 & 6647 & 85,98 \\
\hline TOTAL & 2415 & 100 & 7731 & 100 \\
\hline
\end{tabular}


Foram selecionados os 30 autores mais citados das duas pesquisas. As duas listas apresentam os mesmos 16 autores nas primeiras 30 posições. Este é um importante número a ser avaliado, já que cerca de $50 \%$ dos principais citados nos trabalhos são os mesmos há cerca de dez anos. Quatro autores que estão entre os trinta mais citados nas dissertações não aparecem nos trabalhos da Compós. É interessante observar que Stuart Hall, que é 0 $5^{\circ}$ mais citado nos trabalhos da Compós, não aparece entre os 30 autores mais citados nas dissertações analisadas.

\section{Considerações finais}

Trabalhos de bibliometria podem ser muito úteis para uma auto-compreensão da área. Através da análise de citações é possível observar não apenas as médias de citações e auto-citações, mas também verificar quem são os autores e títulos mais influentes no pensamento comunicacional brasileiro. A reflexão sobre esses padrões de referenciação e da própria autoria (individual ou coletiva) pode inclusive promover novos movimentos e articulações das investigações no país. Mais do que isso, com a continuação de pesquisas deste tipo, estudos comparativos e longitudinais serão possíveis.

Já que esta pesquisa, sobre os artigos da Compós 2008, e a de Maria Immacolata Vassallo de Lopes e Richard Romancini, sobre os trabalhos da Compós 2007, foram conduzidas em paralelo (e, inicialmente, sem que um grupo tivesse conhecimento da pesquisa do outro), ainda não foi possível cruzar os dados levantados. A eventual comparação, mesmo que de padrões de apenas dois anos, já poderá mostrar indícios relevantes da pesquisa de Comunicação no Brasil. É claro, deve-se reconhecer, que estas duas pesquisas tiveram como foco os trabalhos apresentados nos Encontros da Compós, um evento que congrega em sua maioria a produção de professores de programas de pós-graduação. Logo, a comparação da produção discutida em eventos distintos, como Compós e Intercom (este com um grande número de trabalhos em andamento de mestrandos e doutorandos), pode revelar padrões de referenciação distintos, o que se mostra interessante.

0 estudo sobre a remissão a livros, capítulos, periódicos e anais oferece dados relevantes para um melhor entendimento do cenário editorial. 0 próprio sistema de avaliação Qualis poderá se valer desses dados, observando o impacto dos periódicos na área.

Este trabalho, que focou-se especificamente nos padrões de produção e citação dos trabalhos aprovados para a Compós 2008, pôde constatar que 0 esforço de investigação colaborativa, congregando dois ou mais pesquisadores, ainda é muito baixo na área de Comunicação. A maioria dos papers relatam percursos de pesquisa individuais e não de grupos ou parcerias de pesquisa. 
Observou-se uma preocupação em todos os

GTs com uma boa distribuição das referências em relação à escolha dos autores, mantendo um equilíbrio entre trabalhos e autores referenciais na área com produções mais recentes. Por outro lado, apenas 14,1\% das citações referiam-se a textos em periódicos. A grande maioria $(70,7 \%)$ das citações referenciava livros e capítulos.

Quanto aos eventos citados, constatou-se a relevância dos eventos das duas maiores associações de pesquisa da área no Brasil: Compós e Intercom. A primeira obteve 40,6\% das remissões a anais, e a segunda 25,7\%. Vale também reconhecer a penetração dos periódicos dessas instituições, sendo que a E-Compós obteve o número de citações mais expressivo entre todos os periódicos.

Enfim, espera-se que os dados aqui relatados subsidiem futuras pesquisas quantitativas e qualitativas, além de estudos comparativos que mostrem a evolução dos padrões de investigação da área de Comunicação no Brasil.

\section{Referências bibliográficas}

ALVARENGA, Lídia. Bibliometria e Arqueologia do saber de Michel Foucault: traços de identidade teórico-metodológicas. Ciência da Informação, Brasília, v. 27, n. 3, set./dez. 1998.

CARVALHO, Maria Martha. Análises bibliométricas da literatura de química no Brasil. Ciência da Informação, Brasília, v. 4, n. 2, p. 119-141, 1975.
FOWLER, James.H.; AKSNES, Dag.W. Does self-citation pay? Scientometrics, Amsterdam, v. 72, n. 3, p. 427437, 2007.

LANCASTER, Frederick Wilfrid; BURGER, R.H.; RAUCHFUSS, B.M. Use of Literature by East European scientists: what influences place of publication of sources cited? Scientometrics, Amsterdam, v. 24, n. 3, p. 419-439, 1992.

LAWRENCE, Steve. Online or invisible? Nature, v. 411, n. 6837, p.521, 2001.

LOPES, Maria Immacolata Vassallo Lopes; ROMANCINI, Richard. 0 que é uma citação? Análise dos encontros Compós e Intercom 2007. In: Multicom - Intercom - XXXI Congresso Brasileiro de Ciências da Comunicação, 2008, Natal. Anais... Natal: Intercom, 2008.

MEADOWS, Arthur Jack. A Comunicação científica. Brasília: Briquet de Lemos Livros, 1999.

MOED, Henk.Floribert. e outros. Use of bibliometric data for the measurement of university research performance. Research policy, Amsterdam, v. 14, n. 3 , p.131-149, 1985 .

SANZ CASADO, Elías. Manual de estudios de usuarios. Madrid: Fundación Germán Sanches Ruipérez, 1994.

ROSSEAU, Ronald. Indicadores bibliométricos e econométricos para a avaliação de instituições científicas. Ciência da Informação, Brasília, v. 27, n. 2, p.149-158, maio/ago. 1998.

VELHO, Lea. A Contemporaneidade da pesquisa agrícola brasileira como reflexo da distribuição da idade das citações. Ciência da Informação, Brasília, v. 15, n. 1, p.3-9, jan./jul. 1986a.

The meaning of citation in the context of a scientifically peripherical country. Scientometrics, Amsterdam, v. 9, n. 1-2, p. 71-89, 1986b. 
VANZ, Samile Andréa Souza. A produção discente em comunicação: análise das citações das dissertações defendidas nos programas de pós-graduação do Rio Grande do Sul. 2004. Dissertação. (Mestrado em Comunicação e Informação)- Faculdade de Biblioteconomia e Comunicação, Universidade Federal do Rio Grande do Sul, Porto Alegre, 2004. 


\section{Analysis of citing in Compós 2008 papers}

\section{Abstract}

Citing is a procedure used in the production of scientific texts that has the primary function of correlating two texts. As a bibliometric studies tool, the Citation Analysis (CA) allows the correlation between the citer and who is cited, showing the sources that the former used to produce his work. Even though the method has limitations, the Citation Analysis may contribute to the understanding of science structure and development, as well as the behavior of scientists when using information sources. We present a citation analysis of the totality of papers approved in the working groups of the 17th Meeting of the National Association of Graduate Programs in Communication - Compós 2008. From the data collected, the following was analyzed: authors, titles, types of publication (books, chapters, journals, etc.), the most cited journals, collective papers, etc. Among other correlations, we present a parallel between citations to national and foreign authors.

\section{Keywords}

Citation analysis. Compós. Bibliometry. Communication.

\section{Análisis de citas en los trabajo de Compós 2008}

\section{Resumen}

La cita es una práctica usada en la producción de textos científicos que tiene como función primaria enlazar dos textos. Como herramienta de los estudios bibliométricos, el Análisis de Citas (AC) permite relacionar el citador con el citado, mostrando las fuentes que el primero utilizó para producir su trabajo. Aunque presente sus limitaciones, el AC puede ayudar a comprender la estructura y el desarrollo de una ciencia, así como las regularidades de la conducta de los científicos en la utilización de fuentes de información. Este trabajo presenta un análisis de las citas de todos los trabajos aprobados por los Grupos de Trabajo (GT) del XVII Encuentro de la Asociación Nacional de los Programas de Postgrado en Comunicación Compós 2008. Partiendo de los datos recogidos, se busca observar: autores, títulos, tipos de publicación (libros, capítulos, periódicos, etc.), los periódicos más citados, la frecuencia de los trabajos colectivos, etc. Entre otros cruces, se presenta una comparación entre las citas de los autores nacionales y extranjeros.

\section{Palabras clave}

Análisis de citas. Compós. Bibliometría. Comunicación. 


\section{Expediente}

A revista E-Compós é a publicação científica em formato eletrônico da Associação Nacional dos Programas de Pós-Graduação em Comunicação (Compós). Lançada em 2004, tem como principal finalidade difundir a produção acadêmica de pesquisadores da área de Comunicação, inseridos em instituições do Brasil e do exterior.
E-COMPÓS I www.e-compos.org.br I E-ISSN 1808-2599

Revista da Associação Nacional dos Programas de Pós-Graduação em Comunicação. Brasília, v.11, n.3, set./dez. 2008

A identificação das edições, a partir de 2008 passa a ser volume anual com três números.

\section{CONSELHO EDITORIAL}

\section{Afonso Albuquerque}

Universidade Federal Fluminense, Brasil

Alberto Carlos Augusto Klein

Universidade Estadual de Londrina, Brasi

Alex Fernando Teixeira Primo

Universidade Federal do Rio Grande do Sul, Brasil

\section{Alfredo Vizeu}

Universidade Federal de Pernambuco, Brasil

Ana Carolina Damboriarena Escosteguy

Pontifícia Universidade Católica do Rio Grande do Sul, Bras

Ana Silvia Lopes Davi Médola

Universidade Estadual Paulista, Brasil

André Luiz Martins Lemos

Universidade Federal da Bahia, Brasil

Ângela Freire Prysthon

Universidade Federal de Pernambuco, Brasil

Antônio Fausto Neto

Universidade do Vale do Rio dos Sinos, Brasil

Antonio Carlos Hohlfeldt

Pontifícia Universidade Católica do Rio Grande do Sul, Brasil

Arlindo Ribeiro Machado

Universidade de São Paulo, Brasil

César Geraldo Guimarães

Universidade Federal de Minas Gerais, Brasil

Cristiane Freitas Gutfreind

Pontifícia Universidade Católica do Rio Grande do Sul, Brasil

Denilson Lopes

Universidade Federal do Rio de Janeiro, Brasil

Eduardo Peñuela Cañizal

Universidade Paulista, Brasi

Erick Felinto de Oliveira

Universidade do Estado do Rio de Janeiro, Brasil

Francisco Menezes Martins

Universidade Tuiuti do Paraná, Brasil

Gelson Santana

Universidade Anhembi/Morumbi, Brasi

Hector Ospina

Universidad de Manizales, Colômbia

leda Tucherman

Universidade Federal do Rio de Janeiro, Brasil

Itania Maria Mota Gomes

Universidade Federal da Bahia, Brasil

Janice Caiafa

Universidade Federal do Rio de Janeiro, Brasil

Jeder Silveira Janotti Junior

Universidade Federal da Bahia, Brasil

\section{João Freire Filho}

Universidade Federal do Rio de Janeiro, Brasil

John DH Downing

University of Texas at Austin, Estados Unidos

José Luiz Aidar Prado

Pontifícia Universidade Católica de São Paulo, Brasil

José Luiz Warren Jardim Gomes Braga

Universidade do Vale do Rio dos Sinos, Brasil

Juremir Machado da Silva

Pontifícia Universidade Católica do Rio Grande do Sul, Brasil

Lorraine Leu

University of Bristol, Grã-Bretanha

Luiz Claudio Martino

Universidade de Brasília, Brasil

Maria Immacolata Vassallo de Lopes

Universidade de São Paulo, Brasil

Maria Lucia Santaella

Pontifícia Universidade Católica de São Paulo, Brasil

Mauro Pereira Porto

Tulane University, Estados Unidos

Muniz Sodre de Araujo Cabral

Universidade Federal do Rio de Janeiro, Brasil

Nilda Aparecida Jacks

Universidade Federal do Rio Grande do Sul, Brasil

Paulo Roberto Gibaldi Vaz

Universidade Federal do Rio de Janeiro, Brasil

Renato Cordeiro Gomes

Pontifícia Universidade Católica do Rio de Janeiro, Brasil

Ronaldo George Hela

Universidade do Estado do Rio de Janeiro, Brasil

Rosana de Lima Soares

Universidade de São Paulo, Brasil

Rossana Reguillo

Instituto Tecnológico y de Estudios Superiores do Occidente, México

Rousiley Celi Moreira Maia

Universidade Federal de Minas Gerais, Brasil

Sebastião Carlos de Morais Squirra

Universidade Metodista de São Paulo, Brasi

Simone Maria Andrade Pereira de Sá

Universidade Federal Fluminense, Brasil

Suzete Venturelli

Universidade de Brasília, Brasil

Valério Cruz Brittos

Universidade do Vale do Rio dos Sinos, Brasil

Veneza Mayora Ronsini

Universidade Federal de Santa Maria, Brasil

Vera Regina Veiga França

Universidade Federal de Minas Gerais, Brasil
COMISSÃO EDITORIAL

Ana Gruszynski I Universidade Federal do Rio Grande do Sul, Brasil

Rose Melo Rocha I Escola Superior de Propaganda e Marketing, Brasil

\section{CONSULTORES AD HOC}

Alexsandro Galeno Araújo Dantas I Universidade Federal do Rio Grande do Norte, Brasil Isaltina Gomes I Universidade Federal de Pernambuco, Bras João Luís Anzanello Carrascoza I Escola Superior de Propaganda e Marketing, Brasil Malena Segura Contrera I Universidade Paulista, Brasil

Marcia Benetti I Universidade Federal do Rio Grande do Sul, Brasi

Maria Aparecida Baccega I Universidade de São Paulo, Bras

Vander Casaqui I Escola Superior de Propaganda e Marketing, Brasil

Virginia Pradelina da Silveira Fonseca I Universidade Federal do Rio Grande do Sul, Brasil

REVISÃO DE TEXTO E TRADUÇÃo I Everton Cardoso

ASSISTÊNCIA EDITORIAL E EDITORAÇÃO ELETRÔNICA I Raquel Castedo
COMPós I www.compos.org.br

Associação Nacional dos Programas de Pós-Graduação em Comunicação

Presidente

Erick Felinto de Oliveira

Universidade do Estado do Rio de Janeiro, Brasil erickfelinto@uol.com.br

Vice-presidente

Ana Silvia Lopes Davi Médola

Universidade Estadual Paulista, Brasil

asilvia@faac.unesp.br

Secretária-Geral

Denize Correa Araújo

Universidade Tuiuti do Paraná, Brasil

denizearaujo@hotmail.com 